\title{
Gamification and the use of "FPS" or first-person shooting/*seeding* perspective in a laboratory course
}

\section{Dr. Sarah Corinne Rowlinson, University of Florida}

Sarah Rowlinson received the B.S. degree in biomedical engineering from the University of Miami, Coral Gables, FL, USA in 2012, and the Ph.D. degree in bioengineering from Clemson University, Clemson, SC, USA in 2017.

She is a Lecturer and the Undergraduate Coordinator in the J. Crayton Pruitt Family Department of Biomedical Engineering with the University of Florida, Gainesville, FL, USA. In her role as Undergraduate Coordinator, she oversees curriculum development, undergraduate advising, programmatic opportunities, and ABET accreditation for the program's 360 students. As a doctoral student, she studied breast tissue engineering and was an Instructor for the Clemson University General Engineering Program. She also participated in the NSF's Innovation Corps for Learning (I-Corps L) program and was a research mentor through National Science Foundation's Research Experience for Undergraduates (REU) and Research Experience and Mentoring (REM).

Dr. Rowlinson is a member of the American Society for Engineering Education, Biomedical Engineering Society, and Society For Biomaterials. 


\section{Work in Progress: Gamification and the use of "FPS" or first-person shooting/*seeding* perspective in a laboratory course}

This Work-In-Progress paper describes the use of gamification elements for the Cellular Engineering Laboratory course at the University of Florida. Gamification has been emerging as a pedagogical tool over the past few years, for its perceived ability to motivate participants into desired courses of action by making mandatory or mundane tasks rewarding in some way [1]. The application of game design elements in non-gaming environments has been considered as a mechanism to increase motivation, engagement, and attainment of course outcomes [2], [3]. Several common game design elements include Points, Badges, Leader Boards, Avatars, Story Lines, Visualizations, Progress Bars, Punishments, Levels, Challenges, Feedback, and Phrases; all with the intended outcome of facilitating the end-user, or student, experience in the classroom [4].

In this study, we pilot the use of the First-Person Shooter (FPS) perspective to deliver technical content. First-person shooter (FPS) is a video game genre that relies on a first-person perspective; that is, the player experiences the action through the eyes of the protagonist. The player is able to select tools and perform actions to help them on their quest - all through the eyes of the protagonist. In a biomedical engineering wet-laboratory environment, the most common way to teach technical navigation and aseptic technique in a Biological Safety Cabinet (BSC) is one-on-one, with the trainer operating in the BSC and the trainee sitting next to the trainer or behind the trainer's shoulder. This traditional teaching approach is sufficient in research laboratory environments with low student throughput, but does not scale to a course of thirty-two students. As shown in Figure 1, the traditional side-by-side/over-the-shoulder teaching methodology breaks down at a group size as low as six students. At a group size of six, observers experience intermittent obstructed line-of-sight and distracting group cross-talking along with the physical limitation of available space around a BSC. Trainees are not able to easily see the trainer, and therefore subtle techniques required to operate in the BSC environment are not observed or learned. We believe the use of the first-person shooter perspective, or in the case of cell culture "first-person seeding", can be used to improve instructional design and scalability of the course.

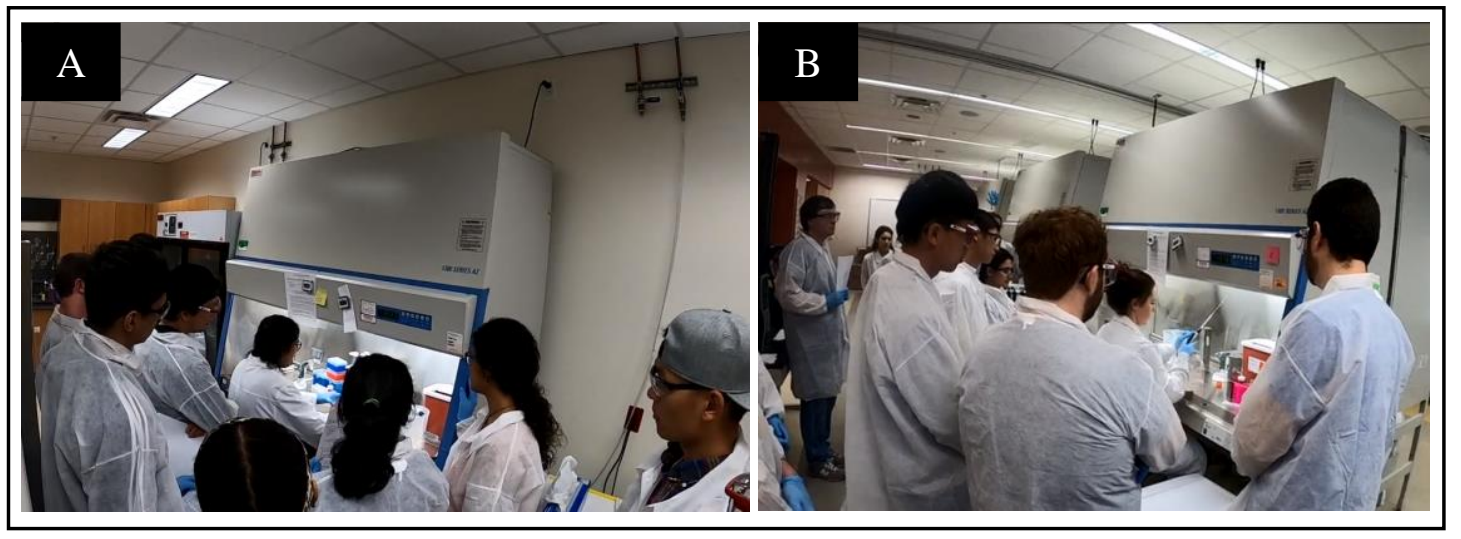

Figure 1: Biological Safety Cabinet environments (A) and (B) depict a group of trainees attempting to observe a trainer operating in the workspace. 


\section{course description and teaching environment}

Cellular Engineering Laboratory is a three-credit, required course that is offered in the fall and spring semesters of junior year. The objective of this course is to provide a hands-on opportunity in maintaining cell cultures and using them as a tool in biomedical research. Together with bioinstrumentation, these courses constitute the dedicated teaching spaces (Figure 2) and fundamental skills that students use later in their course sequence in junior design and senior design. The University of Florida undergraduate biomedical engineering program is capped at one-hundred and twenty students for each graduating cohort, resulting in two sections of $\sim$ thirtytwo students enrolled in the cellular engineering laboratory course each semester.

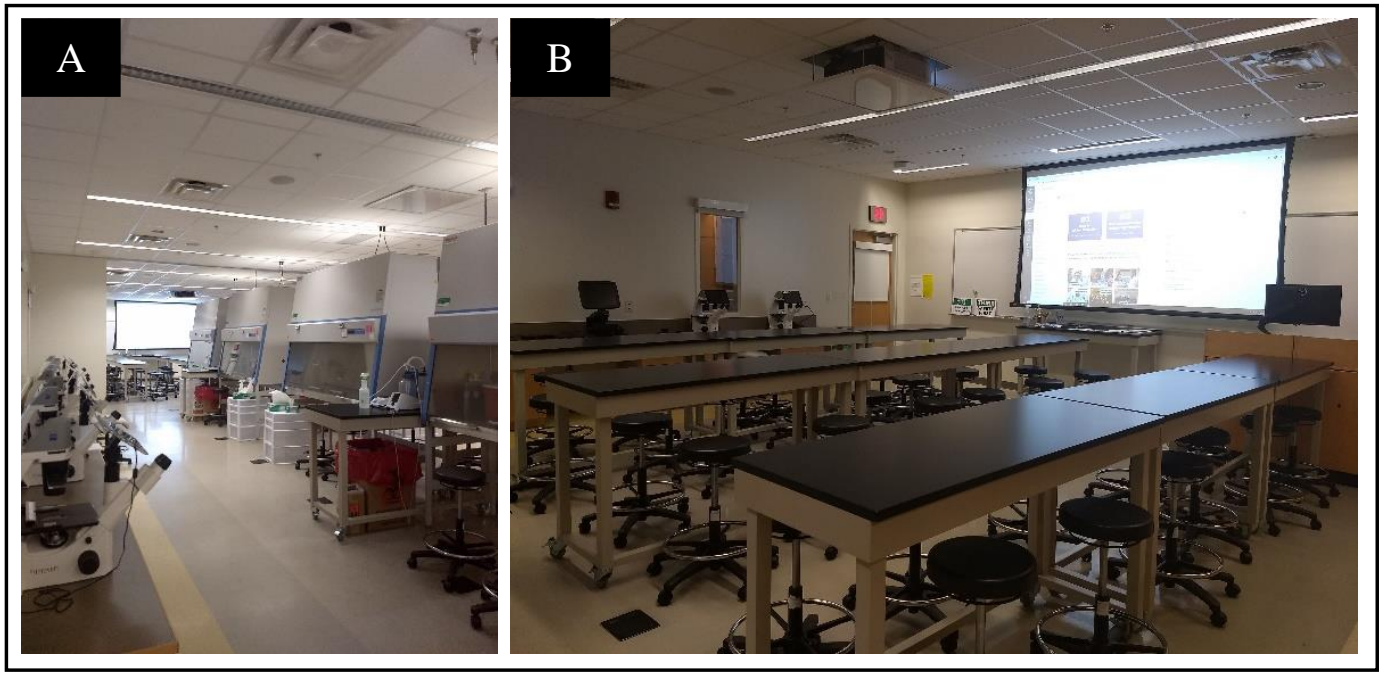

Figure 2: The Cellular Engineering Laboratory has 8 Biological Safety Cabinets, with 4 shown in (A) and the remaining mirrored behind, and a discussion space (B) where all students can see projected images.

For the majority of students enrolled in Cellular Engineering Laboratory, entering this teaching space is their first time navigating in a wet-laboratory environment. Students first learn workplace safety procedures such as biohazardous and chemical waste disposal before operating any equipment. The majority of the semester is then spent introducing students to skills and equipment including aseptic technique, cell culture maintenance, bright-field microscopy, fluorescent staining and microscopy, microplate analysis of cell-based assays, hydrogel fabrication, and Instron mechanical testing. Finally, skills and equipment are critically examined and selected for use in a series of open-ended projects. All equipment mentioned require significant user input and are susceptible to damages that can be costly to fix with repair timelines disruptive to curriculum schedule. Laboratory infrastructure and inventory have a significant impact on course content delivery and management of student training, In some cases there is one device, which can create an equipment-based bottleneck in user training. An example would be students learning how to provide proper software inputs and load their sample into the microplate reader. In other cases there can be a set of devices, with the bottleneck in user training being instructor-based. An example would be students learning how to operate in a biological safety cabinet. As shown in Figure 2-A, there is a significant amount of space an instructor must traverse if they are to provide guidance to students working concurrently in eight workspaces. 


\section{piloting the technology}

Preliminary analysis of student feedback and teaching team (faculty instructor and graduate student teaching assistants) reflections in regard to teaching aseptic technique indicates an opportunity to improve instructional methods. General themes that emerged from the analysis include negative emotions and students reported feeling "frustrated by the sometimes slow pace of the class" and "wanting more immediate feedback." The teaching team members reported feeling "underutilized" and would often facilitate when a student could not hear or see the instructor (the student did not feel comfortable speaking up in order to point out instructor deficit).

The gamified first-person perspective was achieved purchasing a GoPro Hero 8 Black and associated headstrap. A livestream video was established, allowing all thirty-two students in the laboratory discussion area (Figure 2-B) to view actions made by a trainer in the BSC (Figure 3 ). The trainer also wore a microphone connected to the room sound system, ensuring the aseptic technique demonstration was interactive. Students and trainer could ask questions, and the trainer's response could be heard by the entire class. This novel first-person interactive approach is a departure from traditional instructional videos found on research and vendor websites, in which the pre-recorded visuals are acquired using a stationary camera placed behind the shoulder or to the side of the trainer.

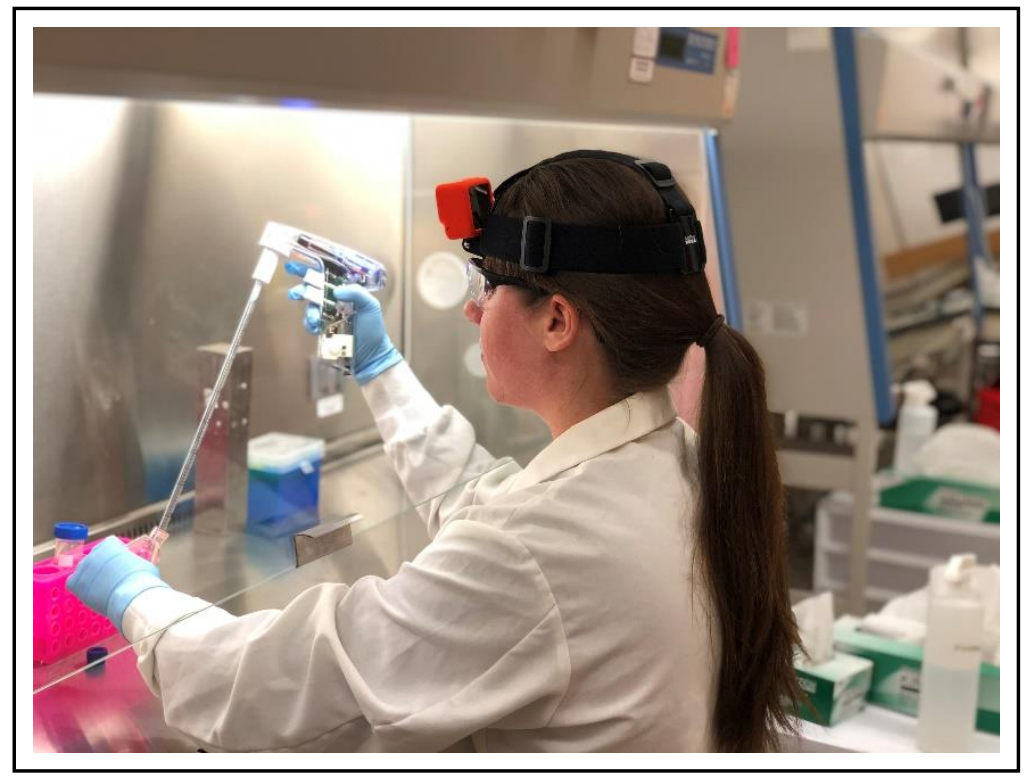

Figure 3: Course instructor wears action camera to create first-person seeding perspective.

\section{future work}

This work represents our initial efforts in using video livestreaming as a tool for teaching aseptic technique and thus, our first attempt at characterizing perspective gamification in the classroom. This initial work has helped us identify other course activities that may benefit from first-person gamification and therefore expand our study sample size. 
To expand on the theme of gamification, other elements under exploration include student "remote" control. In one scenario, students give instructions to the instructor on what to do next. In another scenario, a student would wear the GoPro in the BSC with their peers providing instruction and encouragement. In both scenarios, the cooperative gamification element can be used to engage students towards completing a task. Other elements such as timed races and gameplay critiquing will be explored as low stakes opportunities for competitive play.

The effectiveness of this methodology will be assessed by a combination of quantitative and qualitative measures. Student activity quality and time to completion will be monitored. Students will also complete the College and University Classroom Environment Inventory (CUCEI) to measure their perception of the classroom environment with the addition of the gamification technique. Lab practicum (one-on-one assessment of protocol execution) scores from previous semesters will be compared to semesters where this FPS approach is implemented. Qualitative feedback from students will be collected through open ended survey questions to gain a better appreciation for how the gamification feature impacted their course experience.

\section{motivation and long-term vision}

The equipment used with this gamification feature has implications for future studies, including recordings and livestreams to be used in augmented, mixed, and virtual reality. These recordings could be saved in a repository for future students to access. The repository will start with aseptic technique and will be expanded to include all infrastructure in the teaching laboratory space. Our novel first-person instruction approach has the potential to be used in online learning, flipped classroom, and outreach - and could address barriers to biomedical engineering involvement including access and affordability. At the very least this methodology will facilitate the instructor's effective use of class time, creating the opportunity for time to be repurposed towards new laboratory activities that further diversify students' skillsets.

*Authors Note: In Spring 2020 educators across the world were challenged with the daunting task of converting their courses under very short timeframes to online delivery. It is anticipated that we will also need to deliver our Fall 2020 courses either partially or fully online. The authors look forward to implementing the first-person-seeding perspective and video repository for both in-person interactive and online pseudo-interactive formats. We believe this method of instruction will also facilitate learning laboratory skills while practicing social distancing. 


\section{references}

[1] D. D. Burkey, D. D. Anastasio, and A. Suresh, "Improving Student Attitudes Toward the Capstone Laboratory Course Using Gamification," ASEE Annu. Conf. Exhib., vol. June 23-26, no. Atlanta, GA, 2013.

[2] A. Antonaci, R. Klemke, and M. Specht, "The Effects of Gamification in Online Learning Environments : A Systematic Literature Review," Informatics, vol. 6, no. 32, pp. 1-22, 2019.

[3] R. Alsawaier, "The Effect of Gamification on Motivation and Engagement," Int. J. Inf. Learn. Technol., 2017.

[4] M. R. N. Gari, G. S. Walia, and A. D. Radermacher, "Gamification in Computer Science Education: a Systematic Literature Review,” ASEE Annu. Conf. Exhib., vol. June 24-27, no. Salt Lake City, UT, 2018. 